\title{
Dynamic Strength of Titin's Z-Disk End
}

\author{
Veronika Kollár, ${ }^{1}$ Dávid Szatmári, ${ }^{1}$ László Grama, ${ }^{1}$ and Miklós S. Z. Kellermayer ${ }^{2}$ \\ ${ }^{1}$ Department of Biophysics, Faculty of Medicine, University of Pécs, Szigeti ut 12., Pécs 7624, Hungary \\ ${ }^{2}$ Department of Biophysics and Radiation Biology, Faculty of Medicine, Semmelweis University, Túzoltó u. 37-47., \\ Budapest 1094, Hungary
}

Correspondence should be addressed to Miklós S. Z. Kellermayer, miklos.kellermayer@eok.sote.hu

Received 7 December 2009; Accepted 11 February 2010

Academic Editor: Henk L. M. Granzier

Copyright ( $\odot 2010$ Veronika Kollár et al. This is an open access article distributed under the Creative Commons Attribution License, which permits unrestricted use, distribution, and reproduction in any medium, provided the original work is properly cited.

Titin is a giant filamentous protein traversing the half sarcomere of striated muscle with putative functions as diverse as providing structural template, generating elastic response, and sensing and relaying mechanical information. The Z-disk region of titin, which corresponds to the N-terminal end of the molecule, has been thought to be a hot spot for mechanosensing while also serving as anchorage for its sarcomeric attachment. Understanding the mechanics of titin's Z-disk region, particularly under the effect of binding proteins, is of great interest. Here we briefly review recent findings on the structure, molecular associations, and mechanics of titin's Z-disk region. In addition, we report experimental results on the dynamic strength of titin's Z1Z2 domains measured by nanomechanical manipulation of the chemical dimer of a recombinant protein fragment.

\section{Introduction}

The giant muscle protein titin (also known as connectin) is the third most abundant protein in muscle after actin and myosin, and forms a third myofilament system in skeletal as well as in cardiac muscle [1-3]. Individual titin molecules span half of the sarcomere and run between the Z-disk and the M-line. The main function of titin is to provide a passive mechanical tension in muscle, generating the force responsible for restoring the resting length of the sarcomere [4-7]. In fulfilling its role as a molecular spring it is of great importance that the $\mathrm{N}$ - and $\mathrm{C}$-termini of the molecule be firmly anchored in the Z-disk and the M-line, respectively.

1.1. Titin in the Z-Disk. Immunoelectron microscopic studies revealed that an approximately 800 -residue-long segment at titin's N-terminus is localized within the Z-disk [8]. This region of titin includes the first four immunoglobulin-type domains ( $Z 1$ to $Z 4$ ) and a series of 45-residue repeats, called Z-repeats [9], situated between Z2 and Z3 [10]. While domains $Z 1$ to $Z 4$ are present in all titin isoforms, the number of Z-repeats (up to 7) varies due to extensive differential splicing (Figure 1(a)).
Titin interacts with $\alpha$-actinin via the Z-repeats [11]. The binding more readily detected is found between 7th Zrepeat (Zr7) and the C-lobe of the C-terminal calmodulinlike domain of $\alpha$-actinin. This interaction is similar to that of troponin I to troponin $\mathrm{C}$ and of peptide $\mathrm{C} 20 \mathrm{~W}$ to calmodulin [12]. However, it has been shown that the central Z-repeats are also able to interact with the C-terminal domain of $\alpha$-actinin, and another interaction may occur via a single binding site between titin and the two central spectrin-like repeats of the outermost pair of alpha-actinin molecules [13]. These interactions also enable titin, $\alpha$-actinin and actin to form a ternary complex and contribute to the formation of a stable network structure within the Z-disk.

The thickness and structural properties of Z-disks are largely variable, which was proposed to serve the adaptation of the Z-disk structure to the level of mechanical strain [14]. The observation that the number of Z-repeats in titin varies between species and between muscle types led to the hypothesis that it is the differential expression of titin that accounts for the different thickness and protein composition of Z-disks [9]. However, a direct correlation between the characteristics of $Z$-disks and the expression of different titin isoforms has not been established. 


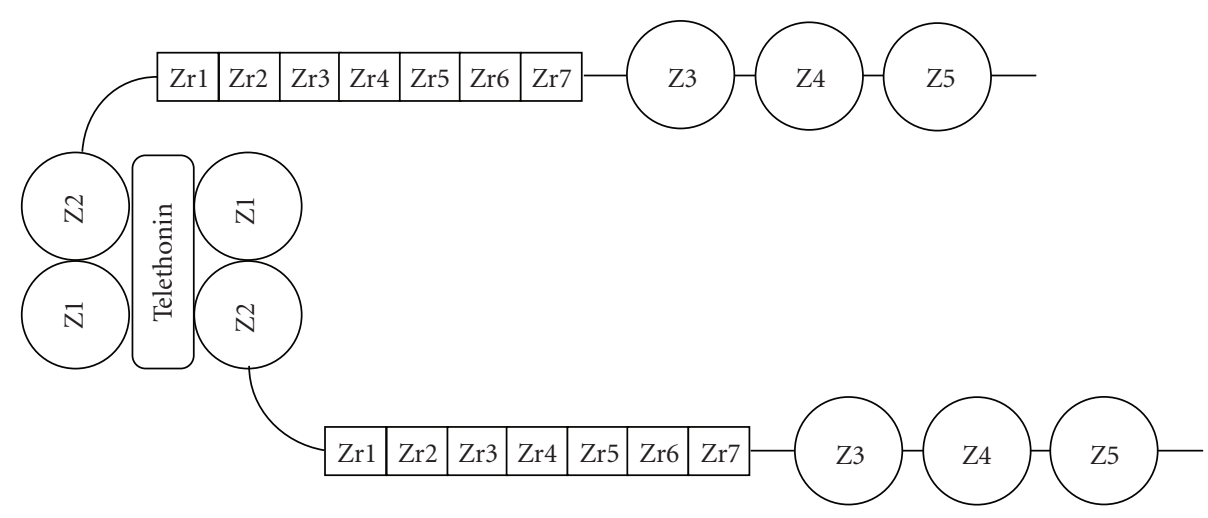

(a)

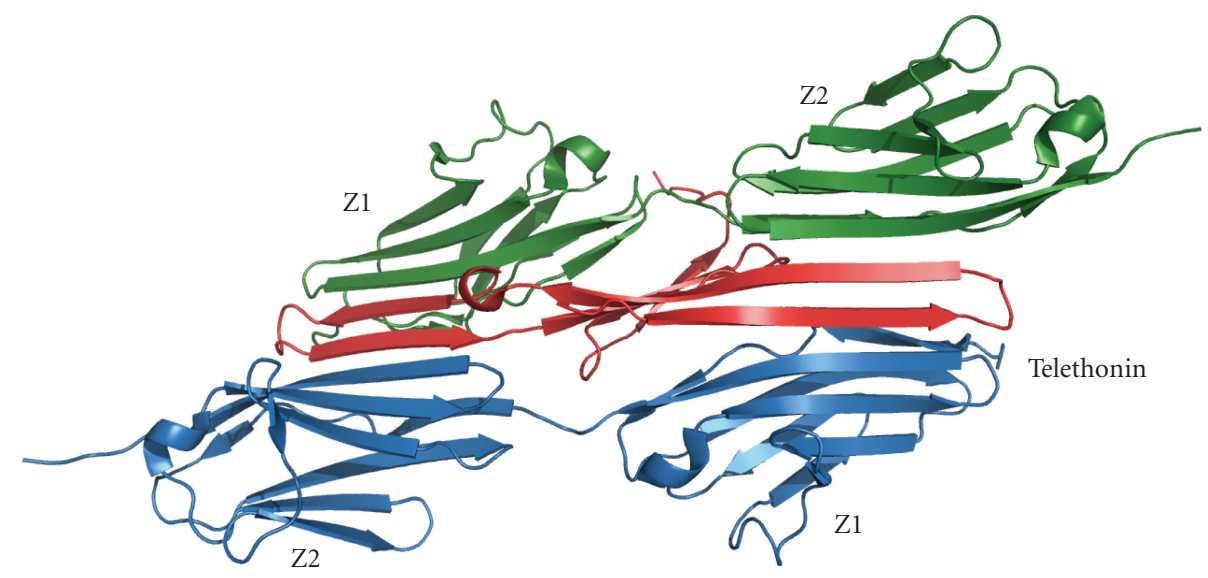

(b)

FIgURE 1: (a) Schematic diagram of the structure of the Z-disk region of titin. (b) Structural model of the Z1Z2-telethonin complex.

1.2. Mechanosensing in the Z-Disk. The Z-disk acts as a link that mechanically integrates contractile and elastic elements, hence it plays a key role in the transmission of active and passive forces. In the last decade, however, new functions of the Z-disk emerged beyond the simple role of a mechanical force transmitter $[15,16]$. As new proteins of the Z-disk were discovered, it became obvious that many of these new components take part in important signaling pathways, many of them having a possible role in stretch sensing.

Stretch is a major factor that is able to induce changes in the morphology and function of muscle. The steps between the onset of mechanical stress and the development of morphological and functional changes include the propagation of the mechanical force to a stress sensor, sensation of stretch, and conversion of the mechanical signal to a biochemical one, finally resulting in alterations of gene expression. This process involves a multitude of molecular players [17].

Titin has been one of the major candidates for the role of the stretch sensor in muscle. Since titin molecules extend in concert with the sarcomere itself, they are well positioned for monitoring the sarcomere's contractile status and possibly transmitting the corresponding mechanical signals. Transduction of the mechanical signal most likely occurs through the interaction of titin with its associated proteins. Different regions ("hot spots") along titin molecules (Z-disk, I-band,
A-band and M-line titin) all participate in a relatively large number of interactions with more than 20 partners known so far [17]. It has been proposed that different regions may sense different parameters: protein complexes in the Z-disk may act as stress sensors that detect and respond to both passive force generated by the titin filaments and active force generated during contraction via the thin filaments, whereas the extensible, I-band region of titin can function as a strain sensor, responding to passive tension alone [18].

1.3. Telethonin Interaction with Z-Disk Titin. One of the most important interactions responsible for anchoring titin in the Z-disk is binding of the titin N-terminus to the Zdisk protein telethonin. Telethonin was identified as a $19 \mathrm{kDa}$ muscle protein present in heart and skeletal muscle. Its transcript is amongst the most abundant in skeletal muscle [19]. Independently, it was discovered as an interacting partner of the titin N-terminus, an interaction that is required for the structural integrity of sarcomere, and the protein was named titin-cap or T-cap [20]. Since its discovery it has been revealed that mutations in the telethonin gene cause limbgirdle muscular dystrophy type $2 \mathrm{G}$, a relatively mild form of autosomal recessive limb-girdle muscular dystrophies [21]. Telethonin has been suggested to be involved in stress sensing, through its interaction with muscle LIM protein 
(MLP) [22]. MLP deficient mice have been shown to develop widened and disorganized Z-disks, while the W4R MLP mutation has been shown to lead to a loss of interaction with telethonin, telethonin mislocalization [22], and has been associated with hypertrophic cardiomyopathy (HCM) $[23,24]$.

Telethonin interacts with the two N-terminal immunoglobulin-like domains of titin (Z1 and Z2), and it colocalizes with the $\mathrm{N}$-terminal part of titin in cultured human skeletal muscle cells [25]. Interestingly, longer titin constructs, including the serine-proline-rich linker region, and the $\mathrm{Z3}$ domain, failed to bind telethonin in yeast two-hybrid assays.

In recent years, the structure of the Z1Z2 doublet and its interaction with telethonin have been subject of numerous investigations. Conformational dynamics studies showed that Z1Z2 preferentially adopts a semiextended conformation with restricted dynamics and a moderately rigid linker that might facilitate the recruitment of its binding partner, telethonin [26]. The term "tertiary structure elasticity" has been introduced for Z1Z2, referring to elasticity resulting from bending and twisting of the domains at intermediate forces, in addition to the entropic elasticity that dominates at low forces and secondary-structure elasticity (unravelling of the domains) that dominates at high forces [27]. X-ray scattering studies on the solution structure of the Z1Z2-telethonin complex indicated a 1:2 association in an antiparallel manner, with telethonin as the central linker [28]. Hence, telethonin was not a simple cap, but rather a possible cross-linker between two titin filaments. The major breakthrough in deciphering the interaction and the building of the complex was solving the structure of the complex by X-ray crystallography (Figure 1(b)) and confirming the antiparallel, palindromic arrangement of the two titin filaments [29]. It is interesting to note that telethonin alone has no stable structure in solution, and it acquires its conformation only upon binding to the Z1Z2 domains.

The cross-linking role of telethonin and the possible mechanical function of the complex raised the question of its mechanical stability. Molecular dynamics simulations performed by Lee et al. predicted an unusually large unfolding force for the complex, in contrast with the relatively low mechanical stability predicted for Z1Z2 alone [30]. The actual strength of the Z1Z2-telethonin bond has been recently measured by stretching the complex using an atomic force microscope (AFM) [31]. The observed dissociation forces were indeed unusually high $(\sim 700 \mathrm{pN})$, far exceeding unfolding forces observed for the Z1Z2 domains alone $(\sim 168 \mathrm{pN})$ or previously measured unfolding forces of other titin Ig-like domains [32]. In addition, Bertz et al., by using protein engineering techniques, demonstrated that the mechanical stability of the complex is sensitive to the pulling direction [30]. Although the forces necessary to unfold Z1Z2 and to disrupt its complex with telethonin have been measured, the stability of Z1Z2 under varying loading rates is not known. Here we explored the dynamic strength of Z1Z2 by exposing the chemical dimer of a recombinant Z1Z2 fragment to a range of mechanical loading rates in nanomechanical experiments.

\section{Materials and Methods}

2.1. Cloning, Expression and Protein Purification. The human skeletal muscle cDNA library was a generous gift of Siegfried Labeit [33]. The nucleotide sequence corresponding to the Z1Z2 titin domain pair was amplified by polymerase chain reaction (PCR). Nucleotide sequence boundaries of the Z1Z2 fragment, based on GenBank accession no. X90568 (version X90568.1, [33]) were 133-717 (aa: 1-195). The fragment was cloned into a pET28a vector (Novagen, Darmstadt, Germany), that carried a kanamycin resistance gene, between NheI and XhoI sites introduced independently with PCR by using specific oligonucleotides. The recombinant protein contained a hexahistidine $\left(\mathrm{His}_{6}\right)$ tag on the N-terminus and two vicinal cysteines on the C-terminus, added to aid subsequent single-molecule manipulation. Escherichia coli BL21(DE3) pLysS cells were transformed with the pET28a-Z1Z2 construct and grown in $2 \times$ YT Microbial EZMix medium (Sigma-Aldrich) in the presence of $30 \mu \mathrm{g} / \mathrm{mL}$ kanamycin until the $\mathrm{OD}_{600}$ of the culture reached 0.6-0.8. Expression was induced by addition of $1 \mathrm{mM}$ isopropylthio$\beta$-D-galactoside and performed for 3 hours at $37^{\circ} \mathrm{C}$. Harvested cells were resuspended in lysis buffer $(50 \mathrm{mM}$ $\mathrm{NaH}_{2} \mathrm{PO}_{4}, 300 \mathrm{mM} \mathrm{NaCl} \mathrm{pH}$ 8.0) supplemented with 0.01 $\mathrm{mg} / \mathrm{mL}$ DNaseI, and were lysed by pulsed sonication (5 minutes, $80 \%$ intensity, Bandelin Sonopuls HD3100). Cell debris was removed by centrifugation at $100,000 \times \mathrm{g}$ for one hour at $4^{\circ} \mathrm{C}$. The supernatant was applied to Talon polyhistidine-Tag purification resin columns (Clontech) equilibrated with lysis buffer. The bound protein was eluted with increasing imidazole concentration series (from $10 \mathrm{mM}$ to $240 \mathrm{mM}$ in lysis buffer). The eluted protein was dyalised against PBS-buffer ( $\mathrm{pH}$ 7.2). Protein concentration was determined spectrophotometrically, using the molar extinction coefficient determined with the ProtParam tool. The electrophoretogram of the purified Z1Z2 fragment is shown on Figure 2(a).

2.2. Single-Molecule Force Spectroscopy. To facilitate the specific binding of the $\mathrm{N}$-terminal, $\mathrm{His}_{6}$-tagged end of the protein to the substrate, in our single-molecule experiments we used glass slides coated with Ni-NTA. Preparation of the slides was described previously [34]. Briefly, cleaned microscope slides were first incubated for 12 hours in toluene vapor containing 2\% Glymo (3-Glycidiloxypropyltrimethoxisylane) (Fluka), then washed with distilled water and dried. Subsequently, the glymo-covered slides were incubated in $0.01 \mathrm{M} \mathrm{Na}_{2} \mathrm{CO}_{3}(\mathrm{pH} 10)$, containing $2 \%$ (wt/vol) N-(5-amino 1-carboxypenthyl)-iminodiacetic acid (NTA) (Dojindo) for 16 hours at $60^{\circ} \mathrm{C}$, then washed with distillated water and dried. Finally, the slides were activated with PBS buffer containing $10 \mathrm{mM} \mathrm{NiCl}_{2}$ and $5 \mathrm{mM}$ glycine ( $\mathrm{pH}$ 8.0) for two hours at room temperature. $\mathrm{Z1Z2}$ fragments were kept in PBS buffer $(137 \mathrm{mM} \mathrm{NaCl}, 2.7 \mathrm{mM} \mathrm{KCl}$, $7.8 \mathrm{mM} \mathrm{Na}_{2} \mathrm{HPO}_{4}, 2.2 \mathrm{mM} \mathrm{KH}_{2} \mathrm{PO}_{4}, \mathrm{pH}=7.3$ ). We created an oxidative environment by omitting DDT from the buffer, to facilitate the formation of Z1Z2 chemical dimers through disulfide bonds between the C-terminal cysteine residues (Figure 2(b)). 


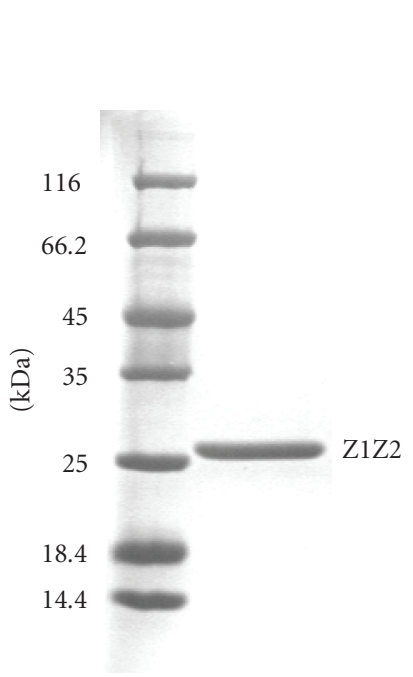

(a)

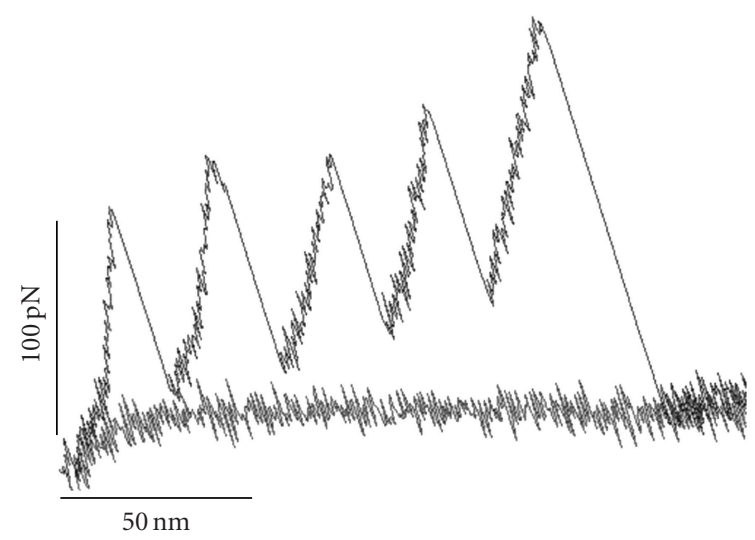

(c)

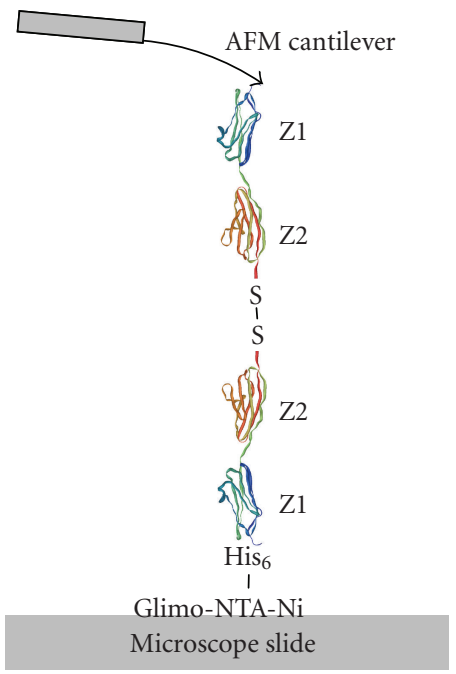

(b)

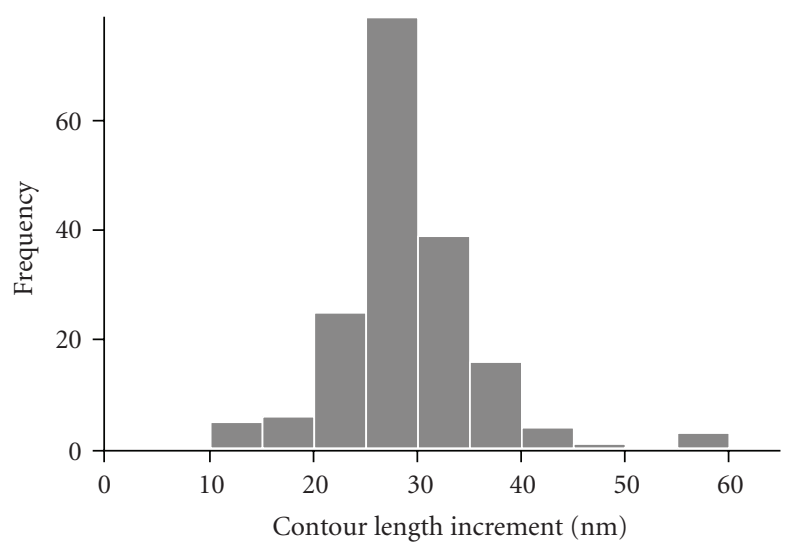

(d)

FIgURE 2: (a) SDS-polyacrylamide electrophoretogram of the recombinant Z1Z2 fragment. (b) Nanomechanical manipulation of the Z1Z2 chemical dimer. The molecular design of the Z1Z2 chemical dimer is indicated. Two recombinant Z1Z2 modules, each with a His ${ }_{6}$-tag at its $\mathrm{N}$-terminus and a cysteine at its C-terminus, respectively, are dimerized via a disulphide bond under oxidizing conditions. The dimer is manipulated by using single-molecule AFM. (c) Force versus end-to-end length curve of a Z1Z2 dimer. The first four sawtooth peaks correspond to the unfolding of each of the four domains (two Z1 and two Z2), and the fifth peak corresponds to the final dissociation of the complex from either the AFM tip or the substrate surface. (d) Distribution of contour-length gain during the sawtooth force transitions. Average contour-length gain per transition was $29.1 \pm 0.6 \mathrm{~nm}( \pm$ SEM).

Molecules were mechanically stretched by using an atomic force microscope (AFM) dedicated for singlemolecule manipulation (MFP1D, Asylum Research, Santa Barbara, CA), mounted on a custom-built, low-profile inverted light microscope. The experimental layout is shown in Figure 2(b). Z1Z2 dimers were allowed to bind to the surface of Ni-NTA-coated glass slides for 10 minutes. Unbound molecules were removed by washing the slide with PBS. The AFM cantilever (Bio-lever, type B; Olympus, Tokyo, Japan) was brought gently in contact with the Ni-NTA-coated substrate, then pulled away from the surface at a constant rate. Stretch rate (cantilever base velocity) was typically $500 \mathrm{~nm} / \mathrm{s}$, or $30,100,300,1000$, and $3000 \mathrm{~nm} / \mathrm{s}$ in pullingspeed-dependent measurements. Compression forces used during the nanomechanical manipulation were between 300 and $700 \mathrm{pN}$. Dwell time on the surface, used to facilitate the binding of a molecule to the tip, was $1 \mathrm{~s}$.

Force versus displacement curves were collected in repeated stretch and release cycles. Force was determined from the bending and stiffness of the cantilever. Cantilever stiffness $(\kappa)$ was obtained by calibration with the thermal method [35]. Typical cantilever stiffness was $\sim 6 \mathrm{pN} / \mathrm{nm}$. The force-displacement curves were corrected for several factors to obtain force versus molecular end-to-end length functions. The zero-length, zero-force data point was obtained from the force response that corresponded to the cantilever tip reaching (or departing from) the substrate surface. Forces $(F)$ were corrected for baseline slope obtained from the force response of the displaced but unloaded cantilever. The end-to-end length $(z)$ of the tethered molecule was 
calculated by correcting the cantilever base displacement $(s)$ with cantilever bending as

$$
z=s-\frac{F}{\kappa} .
$$

2.3. Analysis of Force Data. Force versus molecular endto-end length curves displaying repetitive force peak were further analyzed to obtain unfolding force values for the Z1Z2 construct. The peak force for individual unfolding events in each data set was measured. The last peak, presumably due to the detachment of the molecule either from the tip or from the glass surface, was omitted from analysis. Force peaks associated with a contour-length gain that was significantly different from the value expected based on the length of the unfolded $\mathrm{Z} 1$ or $\mathrm{Z} 2$ domains were also excluded from the analysis. The theoretically expected contour length gain for the $\mathrm{Z} 1$ and $\mathrm{Z} 2$ domains is $32-34 \mathrm{~nm}$, calculated based on the number of residues (101 and 94 for $\mathrm{Z} 1$ and Z2, resp.) and the size of a folded Ig domain $(\sim 4 \mathrm{~nm})$. The relative frequencies of curves (in measurements using $500 \mathrm{pN} / \mathrm{nm}$ pulling speed) with a different number of peaks were the following: 2 peaks: $13 \%, 3$ peaks: $39 \%$, 4 peaks: $30 \%$, 5 peaks: $17 \%$.

Kinetic parameters of Z1Z2 domain unfolding were investigated by two methods: (i) one based on the theory of Bell [36] and Evans and Ritchie [37], and (ii) one using a Monte-Carlo method (see below). The theory of Bell and Evans and Ritchie assumes a single barrier for protein unfolding/refolding. The mean unfolding force depends on $x_{u}$, the distance along the unfolding trajectory in configuration space between the native and transition states, and $k_{u}^{0}$, the unfolding rate constant at zero applied force according to

$$
F=\frac{k_{B} T}{x_{u}} \ln \left(\frac{r x_{u}}{k_{\mathrm{B}} T k_{u}^{0}}\right),
$$

where $r$ is the force loading rate, $k_{B}$ is Boltzmann's constant, and $T$ is absolute temperature. At $300 \mathrm{~K}, k_{B} T=4.14 \mathrm{pNnm}$. The loading rate was calculated by fitting a line to the rising phase of the force trace immediately preceding the unfolding event. Mean unfolding forces were plotted against the loading rate, and fitted with the above function, and $k_{u}^{0}$ and $x_{u}$ were determined from the fit.

2.4. Monte-Carlo Simulation. Forced unfolding of the Z1Z2 titin Ig domains was simulated using a two-state model $[38,39]$. Initially the domains were assumed to be in the folded state. The folding and unfolding rate constants at a given applied force $(F)$ were calculated according to

$$
k_{u / f}=k_{u / f}^{0} \exp \left( \pm \frac{F x_{u / f}}{k_{B} T}\right),
$$

where $u$ and $f$ correspond to unfolding and folding, respectively, and $k_{u / f}^{0}$ is the unfolding/folding rate constant at zero applied force. Negative sign is associated with folding, as force acts against this process. The protein was extended with preset values for $k_{u / f}^{0}, x_{u / f}$ and pulling speed. Force as well as the unfolding/folding rate constants were calculated in small time increments. The force acting on the protein was calculated using the wormlike chain equation:

$$
F=\frac{k_{B} T}{P}\left(\frac{1}{4(1-x / L)^{2}}-\frac{1}{4}+\frac{x}{L}\right),
$$

where $P$ is the persistence length, and $L$ is the contour length of the protein, calculated as $m L_{f}+(n-m) L_{u}$, where $L_{f}$ and $L_{u}$ are the lengths of the folded and unfolded domains, respectively, while $n$ and $m$ are the numbers of total and folded protein regions, respectively. At each extension the probabilities of unfolding and folding were calculated and compared with a randomly generated number. If an unfolding/folding event took place, the contour length $(L)$, the force $(F)$ and the probabilities were recalculated. The parameters $k_{u / f}^{0}$ and $x_{u}$ were varied until the simulated dependence of unfolding force on the pulling speed best fitted the experimental data. To mimic the experimental arrangement as closely as possible and to avoid the effect of chain length, the stretch and release of four-domain-long molecular segments were simulated.

\section{Results and Discussion}

The dynamic stability of the Z-disk region of the giant muscle protein titin was investigated by mechanically manipulating recombinant fragments consisting of titin's Z1 and Z2 domains. Under oxidative conditions, two Z1Z2 domain pairs were assembled via their C-terminal cysteine residues, yielding a 4-domain-long chemical dimer [40-42]. Force versus extension curves measured by stretching the above construct displayed sawtooth patterns, a characteristic of the unfolding of individual domains (Figure 2(c)). Up to four force peaks were observed for the Z1Z2 dimer excluding the last peak, which corresponds to the detachment of the molecule either from the tip or from the glass surface. Data analysis was performed by analyzing the unfolding force as well as the contour length gain. The obtained contour-length gain was $29.1 \pm 0.6 \mathrm{~nm}( \pm$ SEM) (Figure $2(\mathrm{~d}))$. This value is comparable to the theoretically expected contour-length gain $(32-34 \mathrm{~nm})$ calculated based on the number of residues (101 and 94 for Z1 and Z2, resp.) and the size of a folded Ig domain $(\approx 4 \mathrm{~nm})$. Force peaks with contour-length gains significantly different from the expected value were excluded from the analysis.

The distribution of unfolding forces was relatively wide (Figure 3(a)), with an average value of $101 \pm 3 \mathrm{pN}( \pm$ SEM) for a pulling speed of $1000 \mathrm{~nm} / \mathrm{s}$. The unfolding forces observed here are significantly lower than those found by Bertz et al. [31] for a construct in which a single Z1Z2 fragment was flanked by three ubiquitin domains on both the $\mathrm{N}$ - and C-terminal ends. Using this construct Bertz et al. observed an average contour-length gain of $29.4 \pm 0.2 \mathrm{~nm}$ $( \pm$ SEM) [31], which is essentially identical to our results. However, the unfolding forces were $168 \pm 2 \mathrm{pN}$, which far exceed our findings. Even these greater values, however, fall below most of the unfolding forces measured so far 


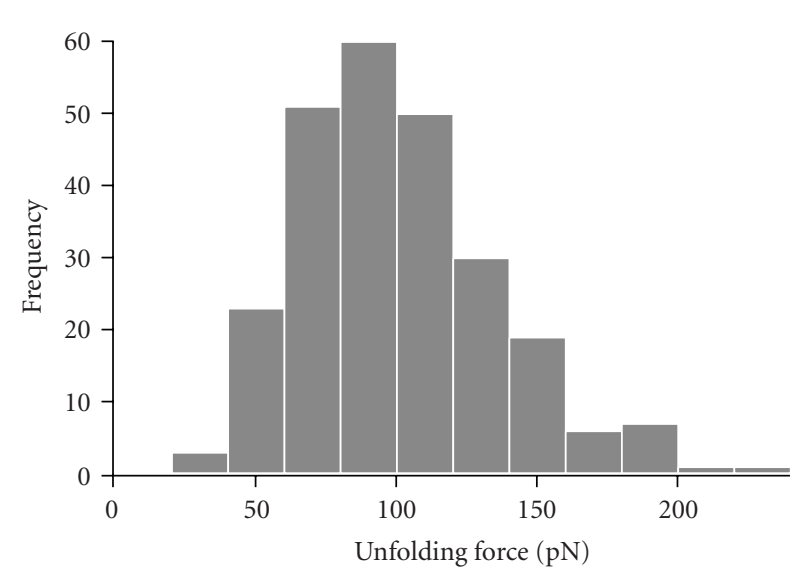

(a)

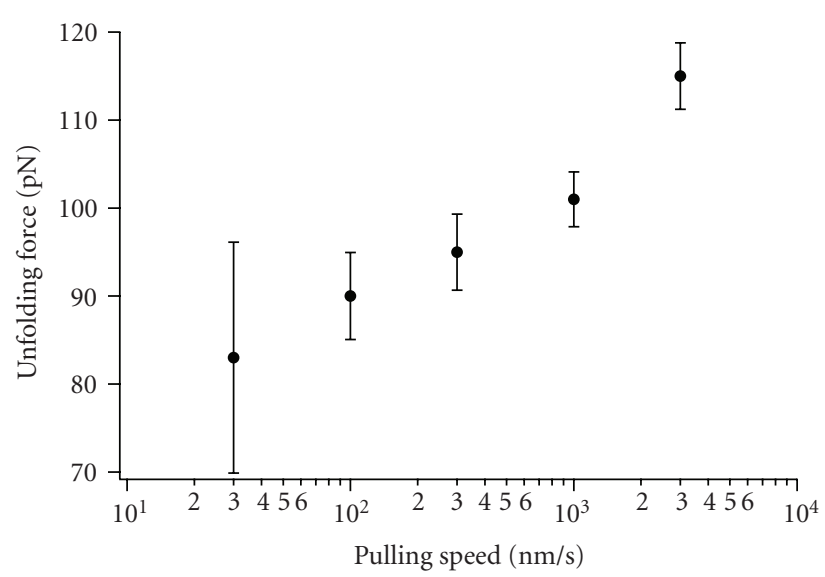

(b)

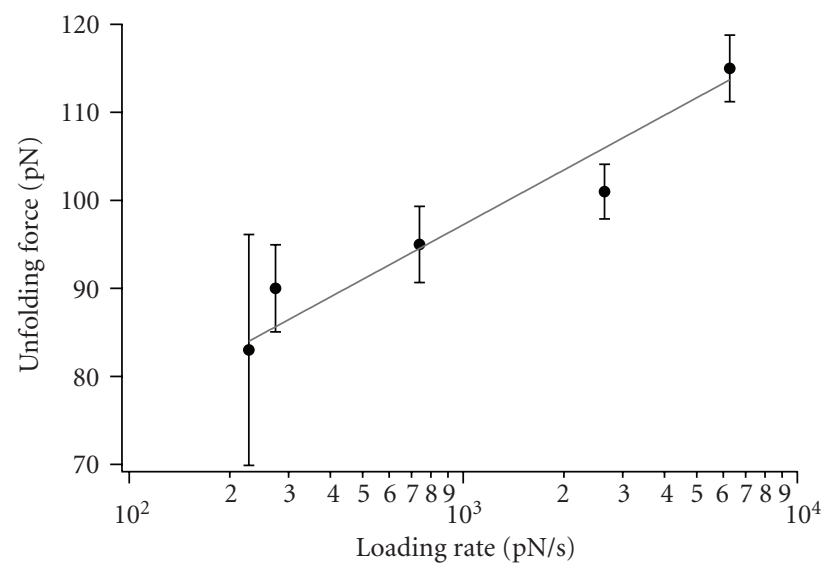

(c)

FIgURE 3: (a) Distribution of unfolding forces of Z1Z2 at $500 \mathrm{~nm} / \mathrm{s}$ pulling speed. (b) Instantaneous unfolding force versus pulling speed. Error bars represent SEM; the average number of data points for the different pulling speeds was 25. (c) Unfolding force versus loading rate. The average number of data points for the different loading rates was 23. Data were fitted with (2).

for an array of titin's globular domains [32], suggesting that the Z1Z2 domains represent a mechanically weak part of titin. We can only speculate about the origin of the difference between our results and the findings of Bertz et al. [31]. It might be possible that the molecular environment imposed on the Z1 and Z2 domains by the recombinant constructs led to either structural stabilization [31] or destabilization (observed here). Although it is generally assumed that recombinant constructs represent well the in situ molecular structure and stability [43], sequence-specific and environmental factors may contribute to an alteration of the dynamic characteristics of the protein. Whatever might be the exact source of variation in structural stability, the Z1Z2 domains seem to be particularly unstable domains within titin.

To obtain an estimate on the kinetic parameters of the unfolding process, we performed the experiments at different pulling speeds ranging from 30 to $3000 \mathrm{~nm} / \mathrm{s}$. The pulling speed dependence of unfolding forces is shown in Figure 3(b). Pulling-speed data were converted to loading rate from the derivative of the force versus extension curves
(Figure 3(c)). By fitting the data with (2), we obtained $8.0 \times 10^{-4} \mathrm{~s}^{-1}$ and $0.52 \mathrm{~nm}$ for the spontaneous unfolding rate $\left(k_{u}^{0}\right)$ and the width of the unfolding potential $\left(x_{u}\right)$, respectively. Using these parameters in a Monte-Carlo simulation, the nanomechanical data were successfully recovered (Figure 4). In contrast to the kinetic parameters found here for Z1Z2, spontaneous unfolding rates of $3.3 \times 10^{-4} \mathrm{~s}^{-1}$ and $2.8 \times 10^{-5} \mathrm{~s}^{-1}$ have been observed for titin's I27 and I28 immunoglobulin domains, respectively [44]. The unfolding potential width was calculated as $0.25 \mathrm{~nm}$ for both of these domains [44]. The spontaneous unfolding rate for the Z1Z2 domains is significantly greater than that of titin's I 27 and I28 domains, indicating faster unfolding and hence lower stability under unloaded conditions. By estimating the shape of the unfolding potential with $x_{u}$, we may explore the dynamic stability of Z1Z2 as well. The $x_{u}$ value for the Z1Z2 fragment is approximately two times larger than that for either I27 or I28. The smaller $x_{u}$ value of I27/I28 indicates that during mechanical unfolding work is done over a very short distance, allowing high forces to be withstood while maintaining structure [45]. By contrast, the large $x_{u}$ of 


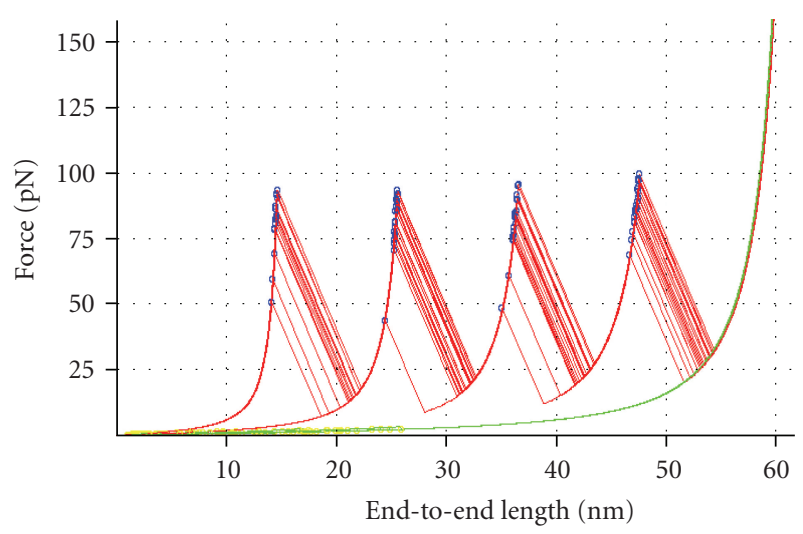

Figure 4: Monte-Carlo simulation of force versus extension data of Z1Z2 using (2) and (3). Data obtained in 15 consecutive stretchrelease cycles at a pulling velocity of $100 \mathrm{nms}^{-1}$ are displayed.

$\mathrm{Z} 1 \mathrm{Z2}$ indicates that work is done over a greater distance, resulting in larger structural changes at relatively low forces. Altogether, the dynamic stability of Z1Z2 is remarkably low.

Our findings indicate that both the thermodynamic stability and the dynamic stability of titin's Z1Z2 domains are very low. Thus, the Z1Z2 domains may not withstand high forces by themselves without severe structural consequences. In the muscle sarcomere, $\mathrm{Z} 1 \mathrm{Z2}$ is tightly associated with telethonin. The extensive network of hydrogen bonds formed between a telethonin molecule and two anti-parallel Z1Z2 domains is thought to result in a structural stabilization so large that the energetics of the Z1Z2-telethonin-Z1Z2 complex become comparable to that of a covalent bond $[29,31]$. We speculate that the primary in vivo function of the strong Z1Z2-telethonin association is the formation of mechanical continuity along the titin-based scaffold of consecutive sarcomeres, and anchorage of titin within the Z-disk might be a secondary function. Mechanically weak domains in titin are also found among the fibronectin domains located in the A-band section of the molecule [46]. Titin's A-band section is thought to be inextensible and therefore structurally stable, conceivably because of tight association with constituents of the thick filament [47]. Tight association with binding partners is thus a common organizing principle in stabilizing mechanically weak regions in titin. Whether and how these nearly static interactions allow structural rearrangements during myofibrillogenesis and sarcomeric protein turnover await further investigation.

\section{Conclusions}

The dynamic stability of titin's Z1Z2 domains was explored here by mechanically manipulating a chemical dimer formed of recombinant protein fragments. Z1Z2 domains have a remarkably low dynamic stability, preventing them from withstanding high mechanical forces regardless of loading rates. It is the association of $\mathrm{Z} 1 \mathrm{Z} 2$ domains with telethonin that provides sufficient structural stabilization so that titin becomes tightly anchored in the Z-disk.

\section{Acknowledgments}

This work was supported by Grants from the Hungarian Science Foundation (OTKA K73256) and the Hungarian National Office of Research and Technology (NANOAMI KFKT-1-2006-0021, OMFB-380/2006) to Miklós S. Z. Kellermayer, and by a grant from the Hungarian Science Foundation (OTKA F49514) to Lászlo Grama.

\section{References}

[1] C. C. Gregorio, H. Granzier, H. Sorimachi, and S. Labeit, "Muscle assembly: a titanic achievement?" Current Opinion in Cell Biology, vol. 11, no. 1, pp. 18-25, 1999.

[2] K. Maruyama, "Connectin/titin, giant elastic protein of muscle," FASEB Journal, vol. 11, no. 5, pp. 341-345, 1997.

[3] K. Wang, "Titin/connectin and nebulin: giant protein rulers of muscle structure and function," Advances in Biophysics, vol. 33, pp. 123-134, 1996.

[4] T. Funatsu, H. Higuchi, and S. Ishiwata, "Elastic filaments in skeletal muscle revealed by selective removal of thin filaments with plasma gelsolin," Journal of Cell Biology, vol. 110, no. 1, pp. 53-62, 1990.

[5] H. L. Granzier and T. C. Irving, "Passive tension in cardiac muscle: contribution of collagen, titin, microtubules, and intermediate filaments," Biophysical Journal, vol. 68, no. 3, pp. 1027-1044, 1995.

[6] R. Horowits, E. S. Kempner, M. E. Bisher, and R. J. Podolsky, "A physiological role for titin and nebulin in skeletal muscle," Nature, vol. 322, no. 6084, pp. 160-164, 1986.

[7] W. A. Linke, M. Ivemeyer, N. Olivieri, B. Kolmerer, J. C. Rüegg, and S. Labeit, "Towards a molecular understanding of the elasticity of titin," Journal of Molecular Biology, vol. 261, no. 1, pp. 62-71, 1996.

[8] H. Yajima, H. Ohtsuka, Y. Kawamura, et al., "A 11.5$\mathrm{kb} 5$-terminal cDNA sequence of chicken breast muscle connectin/titin reveals its Z line binding region," Biochemical and Biophysical Research Communications, vol. 223, no. 1, pp. 160-164, 1996.

[9] M. Gautel, D. Goulding, B. Bullard, K. Weber, and D. O. Fürst, "The central Z-disk region of titin is assembled from a novel repeat in variable copy numbers," Journal of Cell Science, vol. 109, no. 11, pp. 2747-2754, 1996.

[10] M.-L. Bang, T. Centner, F. Fornoff, et al., "The complete gene sequence of titin, expression of an unusual $\approx 700-\mathrm{kDa}$ titin isoform, and its interaction with obscurin identify a novel Zline to I-band linking system," Circulation Research, vol. 89, no. 11, pp. 1065-1072, 2001.

[11] H. Sorimachi, A. Freiburg, B. Kolmerer, et al., "Tissue-specific expression and $\alpha$-actinin binding properties of the Z-disc titin: implications for the nature of vertebrate Z-discs," Journal of Molecular Biology, vol. 270, no. 5, pp. 688-695, 1997.

[12] R. A. Atkinson, C. Joseph, F. Dal Piaz, et al., "Binding of $\alpha$ actinin to titin: implications for Z-disk assembly," Biochemistry, vol. 39, no. 18, pp. 5255-5264, 2000.

[13] P. Young, C. Ferguson, S. Bañuelos, and M. Gautel, "Molecular structure of the sarcomeric Z-disk: two types of titin interactions lead to an asymmetrical sorting of $\alpha$-actinin," The EMBO Journal, vol. 17, no. 6, pp. 1614-1624, 1998.

[14] J. O. Vigoreaux, "The muscle Z band: lessons in stress management," Journal of Muscle Research and Cell Motility, vol. 15, no. 3, pp. 237-255, 1994. 
[15] D. Frank, C. Kuhn, H. A. Katus, and N. Frey, "Role of the sarcomeric Z-disc in the pathogenesis of cardiomyopathy," Future Cardiology, vol. 3, no. 6, pp. 611-622, 2007.

[16] W. G. Pyle and R. J. Solaro, "At the crossroads of myocardial signaling: the role of $\mathrm{Z}$-discs in intracellular signaling and cardiac function," Circulation Research, vol. 94, no. 3, pp. 296305, 2004.

[17] W. A. Linke, "Sense and stretchability: the role of titin and titin-associated proteins in myocardial stress-sensing and mechanical dysfunction," Cardiovascular Research, vol. 77, no. 4, pp. 637-648, 2008.

[18] M. K. Miller, H. Granzier, E. Ehler, and C. C. Gregorio, "The sensitive giant: the role of titin-based stretch sensing complexes in the heart," Trends in Cell Biology, vol. 14, no. 3, pp. 119-126, 2004.

[19] G. Valle, G. Faulkner, A. De Antoni, et al., "Telethonin, a novel sarcomeric protein of heart and skeletal muscle," FEBS Letters, vol. 415, no. 2, pp. 163-168, 1997.

[20] C. C. Gregorio, K. Trombitás, T. Centner, et al., “The NH2 terminus of titin spans the Z-disc: its interaction with a novel $19-\mathrm{kD}$ ligand (T-cap) is required for sarcomeric integrity," Journal of Cell Biology, vol. 143, no. 4, pp. 1013-1027, 1998.

[21] E. S. Moreira, T. J. Wiltshire, G. Faulkner, et al., "Limbgirdle muscular dystrophy type $2 \mathrm{G}$ is caused by mutations in the gene encoding the sarcomeric protein telethonin," Nature Genetics, vol. 24, no. 2, pp. 163-166, 2000.

[22] R. Knöll, M. Hoshijima, H. M. Hoffman, et al., "The cardiac mechanical stretch sensor machinery involves a $\mathrm{Z}$ disc complex that is defective in a subset of human dilated cardiomyopathy," Cell, vol. 111, no. 7, pp. 943-955, 2002.

[23] J. M. Bos, R. N. Poley, M. Ny, et al., "Genotype-phenotype relationships involving hypertrophic cardiomyopathyassociated mutations in titin, muscle LIM protein, and telethonin," Molecular Genetics and Metabolism, vol. 88, no. 1, pp. 78-85, 2006.

[24] C. Geier, A. Perrot, C. Özcelik, et al., "Mutations in the human muscle LIM protein gene in families with hypertrophic cardiomyopathy," Circulation, vol. 107, no. 10, pp. 1390-1395, 2003.

[25] A. Mues, P. F. M. van der Ven, P. Young, D. O. Fürst, and M. Gautel, "Two immunoglobulin-like domains of the Z-disc portion of titin interact in a conformation-dependent way with telethonin," FEBS Letters, vol. 428, no. 1-2, pp. 111-114, 1998.

[26] M. Marino, P. Zou, D. Svergun, et al., “The Ig doublet Z1Z2: a model system for the hybrid analysis of conformational dynamics in Ig tandems from titin," Structure, vol. 14, no. 9, pp. 1437-1447, 2006.

[27] E. H. Lee, J. Hsin, O. Mayans, and K. Schulten, "Secondary and tertiary structure elasticity of titin Z1Z2 and a titin chain model," Biophysical Journal, vol. 93, no. 5, pp. 1719-1735, 2007.

[28] P. Zou, M. Gautel, A. Geerlof, M. Wilmanns, M. H. J. Koch, and D. I. Svergun, "Solution scattering suggests cross-linking function of telethonin in the complex with titin," Journal of Biological Chemistry, vol. 278, no. 4, pp. 2636-2644, 2003.

[29] P. Zou, N. Pinotsis, S. Lange, et al., "Palindromic assembly of the giant muscle protein titin in the sarcomeric Z-disk," Nature, vol. 439, no. 7073, pp. 229-233, 2006.

[30] E. H. Lee, M. Gao, N. Pinotsis, M. Wilmanns, and K. Schulten, "Mechanical strength of the titin Z1Z2-telethonin complex," Structure, vol. 14, no. 3, pp. 497-509, 2006.
[31] M. Bertz, M. Wilmanns, and M. Rief, "The titin-telethonin complex is a directed, superstable molecular bond in the muscle Z-disk," Proceedings of the National Academy of Sciences of the United States of America, vol. 106, no. 32, pp. 1330713310, 2009.

[32] L. Grama, A. Nagy, C. Scholl, T. Huber, and M. S. Z. Kellermayer, "Local variability in the mechanics of titin's tandem Ig segments," Croatica Chemica Acta, vol. 78, no. 3, pp. 405-411, 2005.

[33] S. Labeit and B. Kolmerer, "Titins: giant proteins in charge of muscle ultrastructure and elasticity," Science, vol. 270, no. 5234, pp. 293-296, 1995.

[34] A. Nagy, L. Grama, T. Huber, et al., "Hierarchical extensibility in the PEVK domain of skeletal-muscle titin," Biophysical Journal, vol. 89, no. 1, pp. 329-336, 2005.

[35] J. L. Hutter and J. Bechhoefer, "Calibration of atomic-force microscope tips," Review of Scientific Instruments, vol. 64, no. 7, pp. 1868-1873, 1993.

[36] G. I. Bell, "Models for the specific adhesion of cells to cells," Science, vol. 200, no. 4342, pp. 618-627, 1978.

[37] E. Evans and K. Ritchie, "Dynamic strength of molecular adhesion bonds," Biophysical Journal, vol. 72, no. 4, pp. 15411555, 1997.

[38] M. S. Z. Kellermayer, S. B. Smith, H. L. Granzier, and C. Bustamante, "Folding-unfolding transitions in single titin molecules characterized with laser tweezers," Science, vol. 276, no. 5315, pp. 1112-1116, 1997.

[39] M. Rief, J. M. Fernandez, and H. E. Gaub, "Elastically coupled two-level systems as a model for biopolymer extensibility," Physical Review Letters, vol. 81, no. 21, pp. 4764-4767, 1998.

[40] H. Dietz, M. Bertz, M. Schlierf, et al., "Cysteine engineering of polyproteins for single-molecule force spectroscopy," Nature Protocols, vol. 1, no. 1, pp. 80-84, 2006.

[41] H. Dietz and M. Rief, "Exploring the energy landscape of GFP by single-molecule mechanical experiments," Proceedings of the National Academy of Sciences of the United States of America, vol. 101, no. 46, pp. 16192-16197, 2004.

[42] L. Grama, A. Málnási-Csizmadia, and C. R. Bagshaw, "Mechanical characterization of single green fluorescent protein homopolymer molecules," Biophysical Journal, vol. 82, no. 193a, 2002.

[43] H. Li, W. A. Linke, A. F. Oberhauser, et al., "Reverse engineering of the giant muscle protein titin," Nature, vol. 418, no. 6901, pp. 998-1002, 2002.

[44] H. Li, A. F. Oberhauser, S. B. Fowler, J. Clarke, and J. M. Fernandez, "Atomic force microscopy reveals the mechanical design of a modular protein," Proceedings of the National Academy of Sciences of the United States of America, vol. 97, no. 12, pp. 6527-6531, 2000.

[45] D. J. Brockwell, G. S. Beddard, J. Clarkson, et al., "The effect of core destabilization on the mechanical resistance of 127," Biophysical Journal, vol. 83, no. 1, pp. 458-472, 2002.

[46] M. Rief, M. Gautel, H. E. Gaub, et al., "Unfolding forces of titin and fibronectin domains directly measured by AFM," Advances in Experimental Medicine and Biology, vol. 481, pp. 129-141, 2000.

[47] J. Trinick, "Gytoskeleton titin as a scaffold and spring," Current Biology, vol. 6, no. 3, pp. 258-260, 1996. 

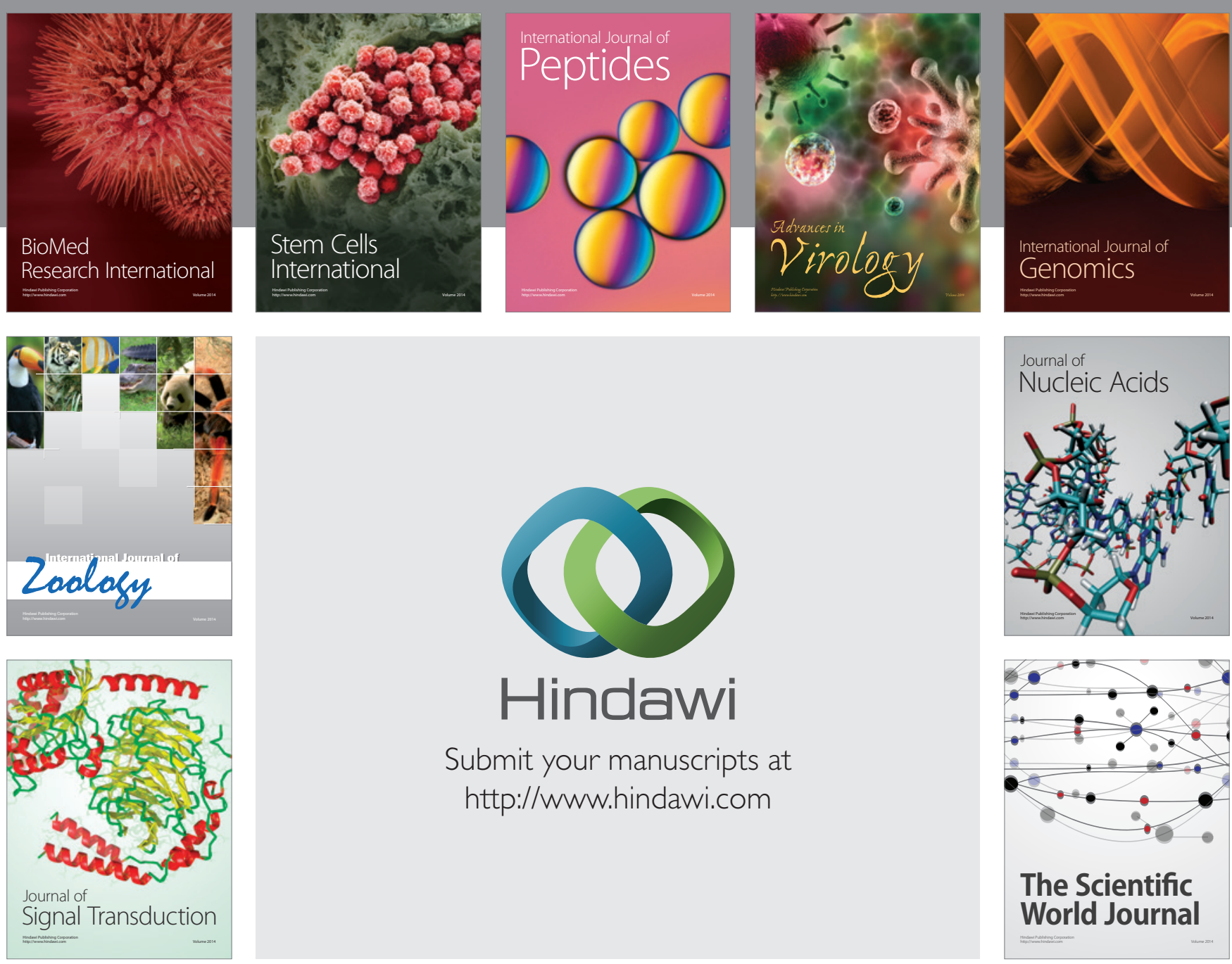

Submit your manuscripts at

http://www.hindawi.com
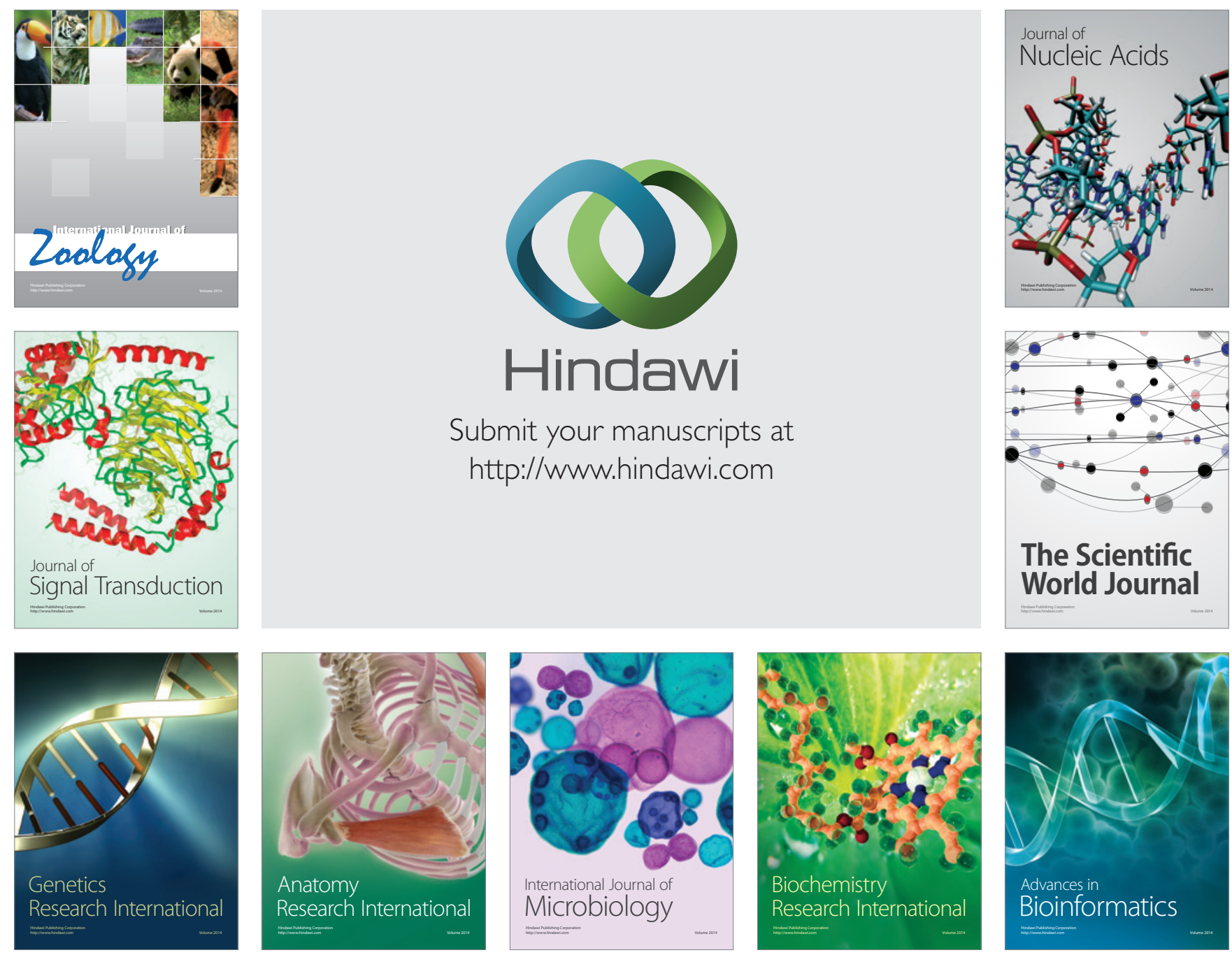

The Scientific World Journal
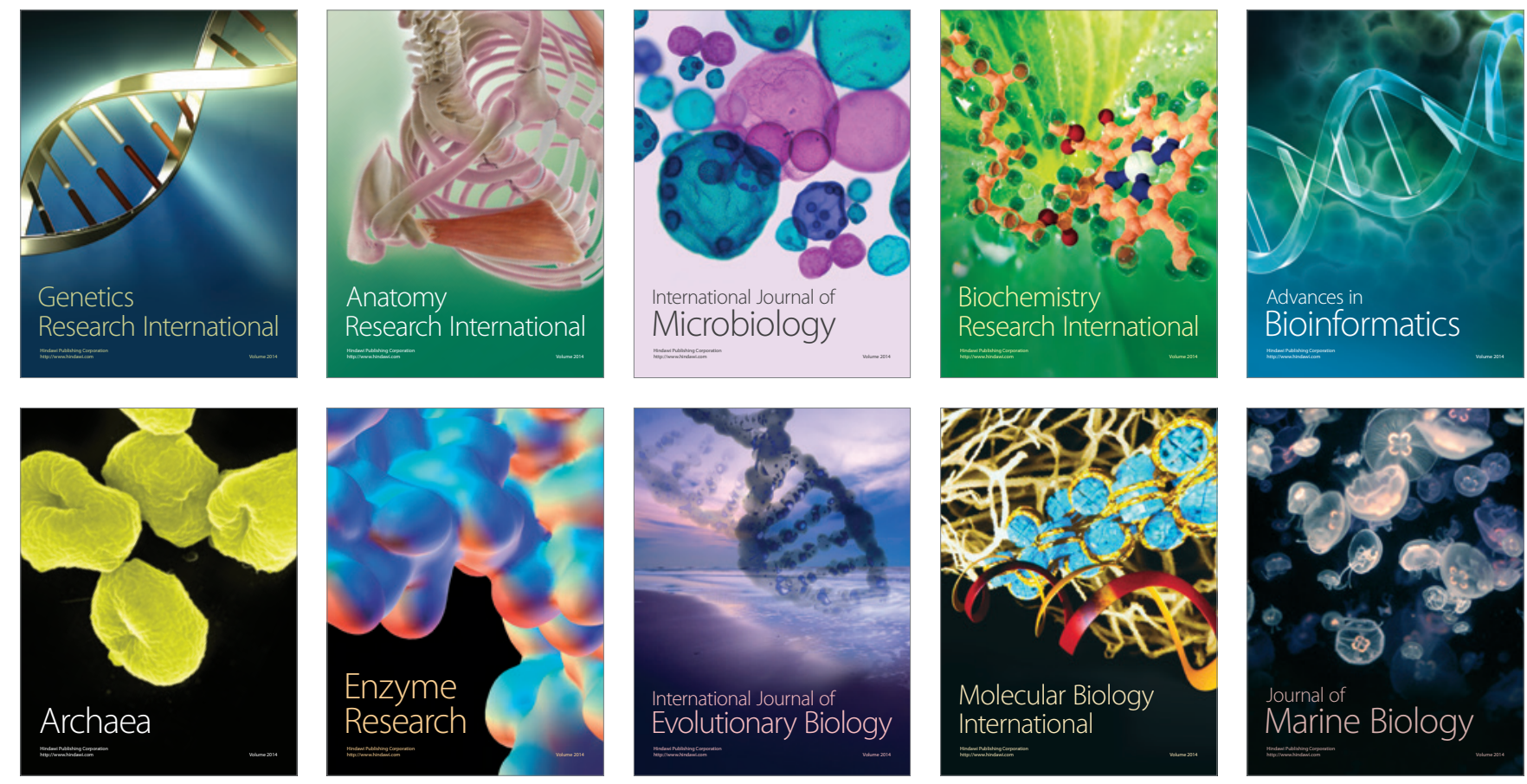